\title{
Stereotactic Radiofrequency Ablation of Breast Cancer Liver Metastases: Short- and Long-Term Results with Predicting Factors for Survival
}

\author{
Peter Schullian $^{1}$ - Edward Johnston ${ }^{2}$ - Gregor Laimer ${ }^{1}$ - Daniel Putzer ${ }^{1}$. \\ Gernot Eberle $^{1} \cdot$ Yannick Scharll $^{1} \cdot$ Claudia Ianetti-Hackl $^{3} \cdot$ Reto Bale $^{1}(\mathbb{D}$
}

Received: 28 October 2020/Accepted: 1 March 2021/Published online: 6 April 2021

(C) The Author(s) 2021, corrected publication 2021

\begin{abstract}
Purpose To evaluate safety, local oncological control, long-term outcome and potential prognostic factors of stereotactic RFA (SRFA) for the treatment of BCLMs.

Methods Between July 2003 and December 2019, 42 consecutive female patients with median age 54.0 years were treated with SRFA at our institution for 110 BCLMs in 48 ablation sessions. Median tumor size was $3.0 \mathrm{~cm}$ (0.8-9.0). Eighteen (42.9\%) patients had extrahepatic metastasis at initial SRFA.

Results Technical success rate was $100 \%$, i.e., all coaxial needles were inserted with appropriate accuracy within $10 \mathrm{~mm}$ off plan and 107/110 (92.3\%) BCLMs were successfully ablated at initial SRFA. Four Grade 1 (8.3\%, $4 / 48)$ and one Grade $2(2.1 \%, 1 / 48)$ complications occurred. No perioperative deaths occurred. Local recurrence developed in 8 of 110 tumors (7.3\%). Overall survival (OS) rates of all patients at 1,3 , and 5 years from the date of the first SRFA were $84.1 \%, 49.3 \%$, and $20.8 \%$ with a median OS of 32.3 months. Univariable cox regression analyses revealed age $>60$ years and extrahepatic disease (without bone only metastases) as significant predictors of worse OS ( $p=0.013$ and 0.025 , respectively). Size and number of
\end{abstract}

Reto Bale

reto.bale@i-med.ac.at

1 Department of Radiology, Section of Interventional Oncology - Microinvasive Therapy (SIP), Medical University of Innsbruck, Anichstr. 35, Innsbruck 6020, Austria

2 Royal Marsden Hospital, 203 Fulham Road, Chelsea, London SW3 6JJ, UK

3 Visceral, Transplant and Thoracic Surgery, Center of Operative Medicine, Medical University of Innsbruck, Anichstr. 35, Innsbruck 6020, Austria metastases, hormone receptor status and time onset did not significantly affect OS after initial SRFA.

Conclusions SRFA is a safe, minimally invasive treatment option in the management of BCLMs, especially in younger patients without advanced extrahepatic metastasis, including those with large liver tumors.

Keywords Radiofrequency ablation - Stereotaxy · Breast cancer liver metastasis

$\begin{array}{ll}\text { Abbreviations } \\ \text { BCLM } & \text { Breast cancer liver metastasis } \\ \text { DFS } & \text { Disease-free survival } \\ \text { LR } & \text { Local recurrence } \\ \text { OS } & \text { Overall survival } \\ \text { SRFA } & \text { Stereotactic radiofrequency ablation }\end{array}$

\section{Introduction}

Breast cancer is one of the most common malignancies in women and is a leading cause of mortality worldwide [1]. Approximately, $20 \%$ of breast cancer patients develop metastatic disease [2], with the lungs, liver, bone and brain being the most common sites. Breast cancer liver metastasis (BCLM) confer a poor prognosis of 4-8 months survival [3] and are found in approximately $50 \%$ of patients with metastatic disease, where $5-12 \%$ of patients have liver only metastases [3]. However, patients with negative resection margins after surgery show 5-year survival rates up to $40 \%$ [4]. 
Advanced breast cancer is primarily treated by systemic hormone therapy and/or chemotherapy, and despite advancements in systemic treatment, median overall survival and 5-year survival rates remain low, at 18-24 months and 27\%, respectively [5].

Recent studies suggest that subgroups of breast cancer patients with oligometastatic disease benefit from additional locoregional treatment $[6,7]$, which is defined by the 3rd ESO-ESMO (European School of Oncology-European Society for Medical Oncology) consensus guidelines as limited metastatic disease with up to five metastases that are potentially amenable for local treatment [8].

Percutaneous thermal ablation methods, such as radiofrequency ablation (RFA) and microwave ablation (MWA) have gained widespread acceptance as a minimally invasive treatment option in the management of primary and metastatic liver tumors [9-12]. Despite several studies showing promising results for RFA in BCLM [13, 14], high-quality evidence is still lacking.

Stereotaxy (derived from the Greek meaning "solid arrangement"), allows the planning of complex trajectories using three-dimensional image datasets with precise transformation into real patients using a Cartesian coordinate system [15]. Furthermore, fusion with previously acquired MR images in case of poor tumor visibility, immediate post-ablation contrast-enhanced CT fusion with the planning CT for reliable assessment of ablation results, allows more complex interventions such as large tumors and those in challenging localizations such as the hepatic dome or caudate lobe.

The purpose of the present study was to evaluate safety, local oncological control, long-term outcome and potential prognostic factors of stereotactic RFA (SRFA) for the treatment of BCLMs.

\section{Materials and Methods}

\section{Patient Selection}

The local institutional review board approved this retrospective single-center study, and all patients included gave their informed consent. Each case was reviewed and the treatment plan was approved by consensus in multidisciplinary tumor advisory board meetings.

One thousand seven consecutive patients were treated by SRFA between July 2003 and December 2019. Twentyeight patients who underwent SRFA for benign liver tumors were excluded. Forty-two consecutive patients with BCLM were treated in 48 ablation sessions and included in the study. Table 1 shows the baseline characteristics of the study group.
Table 1 Patient characteristics of 42 patients with 110 breast cancer liver metastasis undergoing 48 SRFA for local treatment

\begin{tabular}{ll}
\hline Patient characteristics & Study Gr. \\
\hline Age, median years (range) & $54.0(31-82)$ \\
Sex (female/male), $n(\%)$ & $42 / 0(100 / 0)$ \\
Tumor size, median (range) & $3.0 \mathrm{~cm}(0.8-9.0)$ \\
Tumor number at begin, $n$ (range) & $2(1-8)$ \\
$n=1, n(\%)$ & $16(38.0)$ \\
$n=2, n(\%)$ & $13(31.0)$ \\
$n \geq 3, n(\%)$ & $13(31.0)$ \\
Coaxial needles per session, $n$ (range) & $9(3-16)$ \\
Time onset of BCLM, median months (range) & $30.5(0-136)$ \\
Extrahepatic metastasis before SRFA & $18(42.9)$ \\
Bone, $n(\%)$ & $6(14.3)$ \\
Bone and lung $n(\%)$ & $4(9.5)$ \\
Lung, $n(\%)$ & $4(9.5)$ \\
Lymph nodes, $n(\%)$ & $1(2.4)$ \\
Bone and lymph nodes, $n(\%)$ & $3(7.1)$ \\
Treatment before SRFA & \\
CTX, $n(\%)$ & $19(45.2)$ \\
HT, $n(\%)$ & $2(4.8)$ \\
Combined CTX and HT, $n(\%)$ & $17(40.5)$ \\
Combined CTX, HT and HR, $n(\%)$ & $4(9.6)$ \\
Treatment after SRFA & \\
CTX, n (\%) & $18(42.9)$ \\
HT, n (\%) & $2(4.8)$ \\
Combined CTX and HT, $n(\%)$ & $18(42.9)$ \\
\hline$B C L M ~$ & \\
&
\end{tabular}

$B C L M$ breast cancer liver metastasis, SRFA stereotactic radiofrequency ablation, $H R$ hepatic resection, $C T X$ chemotherapy, $H T$ hormone therapy, $c R F A$ conventional RFA

Exclusion criteria for SRFA comprised (i) platelet count $<50,000 / \mathrm{mm} 3$ (ii) prothrombin activity $<50 \%$ and (iii) tumor location close to $(<10 \mathrm{~mm})$ the central bile ducts. Tumor diagnosis was confirmed by multiphasic contrast MRI or CT and inconclusive cases were validated by biopsy.

\section{SRFA Procedure}

The method of SRFA has been reported in detail previously [16-18]. An example SRFA for BCLM is shown in Fig. 1.

\section{Outcome Measurements}

Sufficiently precise coaxial needle placement (deviation from plan $<1 \mathrm{~cm}$ at each needle tip) was defined as a technical success. Technical efficacy and local recurrence rate (LR) were determined by contrast-enhanced CT or MR follow-up examinations performed at intervals of 1 month 
Fig. 1 A 50-year-old female with breast cancer liver metastases measuring up to $3.0 \mathrm{~cm}$. A Contrast enhanced CT-image of one BCLM (black arrowhead) in segment II/III close to the stomach. B Contrast enhanced CT-image showing the other two subcapsular BCLMs in the right lobe (black arrowhead). C Maximum Intensity Projection image of the control CT with 12 coaxial needles in place. D Fused images of the control CT with the planning CT showing superposition of planned paths with inserted coaxial needles. E, F Black arrowheads

demonstrating ablation zones on CT images immediately after the procedure and after 36 months later without evidence of local recurrence
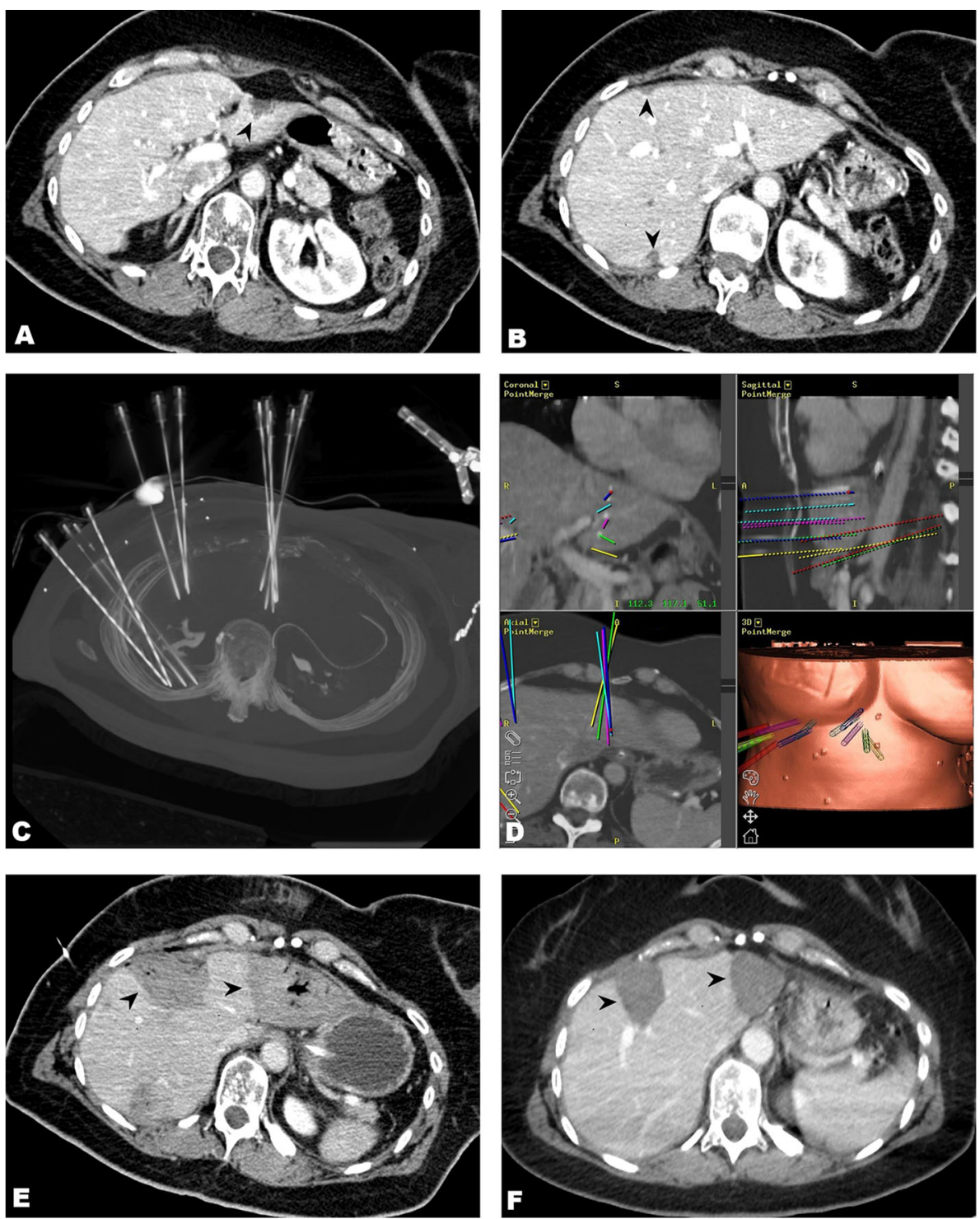

and 3 months after SRFA. Images were evaluated in consensus by two experienced abdominal radiologists (radiologist 1 with 20 years of experience and radiologist 2 with 11 years of experience). Primary technical efficacy rate was evaluated for each tumor, defined as the absence of residual disease on the 1-month follow-up CT. Secondary technical efficacy rate was defined as tumors requiring repeat ablation due to residual tumor. Appearance of new nodules within or immediately adjacent to ablation zones or the original tumor was defined as LR. New nodules distant to the ablation zone and / or the original tumor were defined as distant tumor recurrence. Complications were defined according to the CIRSE Classification System for Complications [19]. Disease-free (DFS) and overall survival (OS) were calculated from the date of initial SRFA until the date of death due to malignancy or other causes (i.e., event), until date of relapse (DFS) or until the last follow-up visit (i.e., censoring).

\section{Prognostic Factors}

Age $(>/<60$ years), tumor size $(>/ \leq 3 \mathrm{~cm})$, tumor number $(>/=1)$, tumor distribution (uni-/bilobar), presence of extrahepatic disease, time from primary tumor to BCLM $(>/<24$ months) and hormone receptor status were evaluated as potential prognostic factors for OS.

\section{Statistics}

Statistical analysis was performed using IBM-SPSS v24. Data were expressed as total numbers, median and range. Differences between categorical variables were evaluated using the $X^{2}$ test and between independent continuous 
variables using the Mann-Whitney U test. OS and DFS were evaluated using the Kaplan-Meier method. Cox regression was used to analyze potential factors of OS. The variables of interest $(p<0.1)$ identified in the univariable analysis were further analyzed in a multivariable analysis using the cox regression model. $p<0.05$ was considered statistically significant.

\section{Results}

\section{Patient Characteristics}

In the study group, 42 females with a total of 110 BCLMs had a median age of 54.0 (31.0-82.0). Median tumor size was $3.0 \mathrm{~cm}(0.8-9.0)$, and a median of 2 BCLM (1-6) were treated per ablation session (48 sessions in total). At baseline (initial SRFA), 16 (38.0\%) patients had a solitary liver metastasis, 13 (31.0\%) had two metastases, and 13 (31.0\%) patients had three or more liver metastases. BCLMs were synchronous metastases in $7(16.7 \%)$ patients and metachronous in $35(83.3 \%)$. The median time between primary diagnosis and detection of BCLM was 30.5 months (range 0-136 months). Eighteen (42.9) patients had extrahepatic metastasis, with $6(14.3 \%)$ in bone, 4 (9.5\%) in bone and lung, 4 (9.5\%) in lung, 1 (2.4\%) in lymph nodes and $3(7.1 \%)$ in bone and lymph nodes. Before SRFA, 19 (45.2\%) patients underwent chemotherapy, $2(4.8 \%)$ hormone therapy, $17(40.5 \%)$ combined treatment of chemotherapy and hormone therapy and 4 (9.6\%) a combined treatment of chemotherapy, hormone therapy and hepatic resection. No patient received immunotherapy. After SRFA, 18 (42.9\%) patients received chemotherapy, $2(4.8 \%)$ hormone therapy and 18 (42.9\%) a combination of hormone and chemotherapy. See Table 1 for details.

During ablation, BCLMs were confirmed histologically in 18 of $42(42.3 \%)$ patients. In one patient the result was inconclusive due to insufficient histological material. Of the remaining 23 patients, BCLMs were confirmed histologically before ablation in 20 patients, and no histological result was available in 3 patients.

\section{Periprocedural Complications}

According to the CIRSE Classification System for Complications, four Grade $1(8.3 \%, 4 / 48)$ and one Grade 2 $(2.1 \%, 1 / 48)$ complications occurred. Four patients developed arterial bleeding from subcapsular liver vessels, managed by transarterial coil embolization in the same anesthetic session. One major pleural effusion required treatment with a chest tube. Median hospital stay after
SRFA was 4.5 days, ranging from $2-39$ days. No perioperative deaths occurred.

\section{Local Tumor Control and Distant Tumor Progression}

Technical success rate was $100 \%$, i.e., all coaxial needles were inserted with appropriate accuracy within $10 \mathrm{~mm}$ off plan. 107/110 liver metastases were successfully ablated at initial SRFA (97.3\% primary technical efficacy rate), whereby 1 of 3 tumors were successfully treated in a second session, resulting a secondary technical efficacy rate of $98.2 \%$. Local recurrence (LR) developed in 8 of 110 tumors $(7.3 \%)$ after a median imaging follow-up of 10.9 months (range 1.4-112 months). Details for insufficient local control are presented in Table 2. An overview of success rates is provided in Table 3.

During follow-up, 19/42 (45.2\%) patients developed disease-progression, with 12 (28.6\%) developing multiple new liver metastases, $3(7.1 \%)$ developing extrahepatic metastases and 8 patients $(19.0 \%)$ both multiple new liver metastases and extrahepatic metastases.

There was no significant difference in LR for lesions $>3 \mathrm{~cm}$ and $<3 \mathrm{~cm}$ with LR rates of $11.5 \%(3 / 26)$ and $6.0 \%(5 / 84)$, respectively $(p=0.338)$. A tumor location close to organs (LR $11.1 \%, 1 / 9$ vs. all other locations with $6.9 \%, 7 / 101 ; p=0.644)$, close to the liver capsule (LR $8.3 \%, 3 / 36$ vs. all other locations with $6.8 \%, 5 / 74$; $p=0.765$ ), close to the diaphragm (LR $10.0 \%, 1 / 10 \mathrm{vs.} \mathrm{all}$ other locations with $7.0 \%, 7 / 100 ; p=0.728$ ) or to large vessels (LR $11.1 \%, 2 / 18$ vs. all other locations with $6.5 \%$, $6 / 92 ; p=0.493$ ) did not significantly affect LR.

\section{Overall and Disease-Free Survival (Figure 2 and Figure 3)}

Overall survival (OS) rates of all patients at 1,3 , and 5 years from the date of the first SRFA were $84.1 \%, 49.3 \%$, and $20.8 \%$ with a median OS of 32.3 months $(95 \% \mathrm{CI}$ 20.6-50.3). The corresponding OS rates at 1,3 , and 5 years from the date of tumor diagnosis were $97.6 \%, 87.8 \%$, and $72.3 \%$ with a median OS of 93.2 months $(95 \%$ CI $65.6-120.8$ ) and $92.5 \%, 62.6 \%$, and $35.9 \%$ with a median OS of 48.2 months (95\% CI 39.9-56.5) from the date of liver metastasis diagnosis.

Univariable cox regression analyses revealed age $>60$ years $(p=0.013$, HR 2.3 CI $0.9-6.0)$ and extrahepatic disease (excluding patients with bone only metastasis; $p=0.025$, HR 1.8 CI 0.7-5.1) as significant predictors of worse OS. Median OS in BCLM patients without extrahepatic disease at initial SRFA was 41.8 months with OS rates at 1, 3, and 5 years of $89 \%, 59 \%$ and $27 \%$. By comparison, BCLM patients younger than 60 years showed 
Table 2 Details of local treatment failures after SRFA

\begin{tabular}{|c|c|c|c|c|c|c|c|}
\hline ID & Age & Size $(\mathrm{cm})$ & Needles & Ablation time (min) & Segment & Location properties & Outcome \\
\hline 1 & 51 & 3.0 & 5 & 48 & $\mathrm{IVb}$ & $\mathrm{gb}$ & $\mathrm{iA}$ \\
\hline 2 & 44 & 7.0 & 8 & 80 & VI, VII & $\mathrm{v}$ & $\mathrm{iA}$ \\
\hline 3 & 45 & 3.0 & 5 & 40 & IVa & $\mathrm{sc}$ & $\mathrm{iA}$ \\
\hline 4 & 63 & 6.5 & 9 & 51 & $\mathrm{~V}, \mathrm{VI}$ & - & LR \\
\hline 5 & 50 & 5.5 & 8 & 54 & III, IVb & $\mathrm{v}, \mathrm{sc}$ & LR \\
\hline 6 & 70 & 2.0 & 2 & 16 & II & $\mathrm{sp}$ & LR \\
\hline 7 & 70 & 2.4 & 2 & 16 & IVb & $\mathrm{sc}$ & LR \\
\hline 8 & 70 & 1.8 & 2 & 12 & IVb & - & LR \\
\hline 9 & 70 & 1.0 & 1 & 12 & II & $\mathrm{v}$ & LR \\
\hline 10 & 45 & 2.0 & 3 & 32 & III & o & LR \\
\hline 11 & 51 & 6.2 & 13 & 70 & VI, VII & $\mathrm{sc}$ & LR \\
\hline
\end{tabular}

SRFA stereotactic radiofrequency ablation, $v$ close to major vessel, $s c$ subcapsular, $s p$ subphrenic, $o$ close to organ, $g b$ close to gallbladder, $i A$ incomplete ablation, $L R$ local recurrence
Table 3 Tumor-based therapy success rates

\begin{tabular}{ll}
\hline Rate & \\
\hline Technical success, $n(\%)$ & $110 / 110(100)$ \\
Primary technical efficacy, $n(\%)$ & $107 / 110(97.3)$ \\
Secondary technical efficacy, $n(\%)$ & $108 / 110(98.2)$ \\
Local recurrence, $n(\%)$ & $8 / 110(7.3)$
\end{tabular}

better survival with a median OS 41.8 and OS rates at 1,3 , and 5 years of $92 \%, 63 \%$ and $30 \%$. Size and number of BCLM, hormone receptor status and time onset of BCLM did not significantly affect OS after initial SRFA. Full details are provided in Table 4.

Multivariable cox regression analyses showed no independent prognostic factors for OS. Disease-free survival (DFS) for all patients at 1,3, and 5 years from the date of the first SRFA were $45.3 \%, 22.3 \%$, and $15.9 \%$ with a median OS of 10.5 months (95\% CI 6.8-25.0).

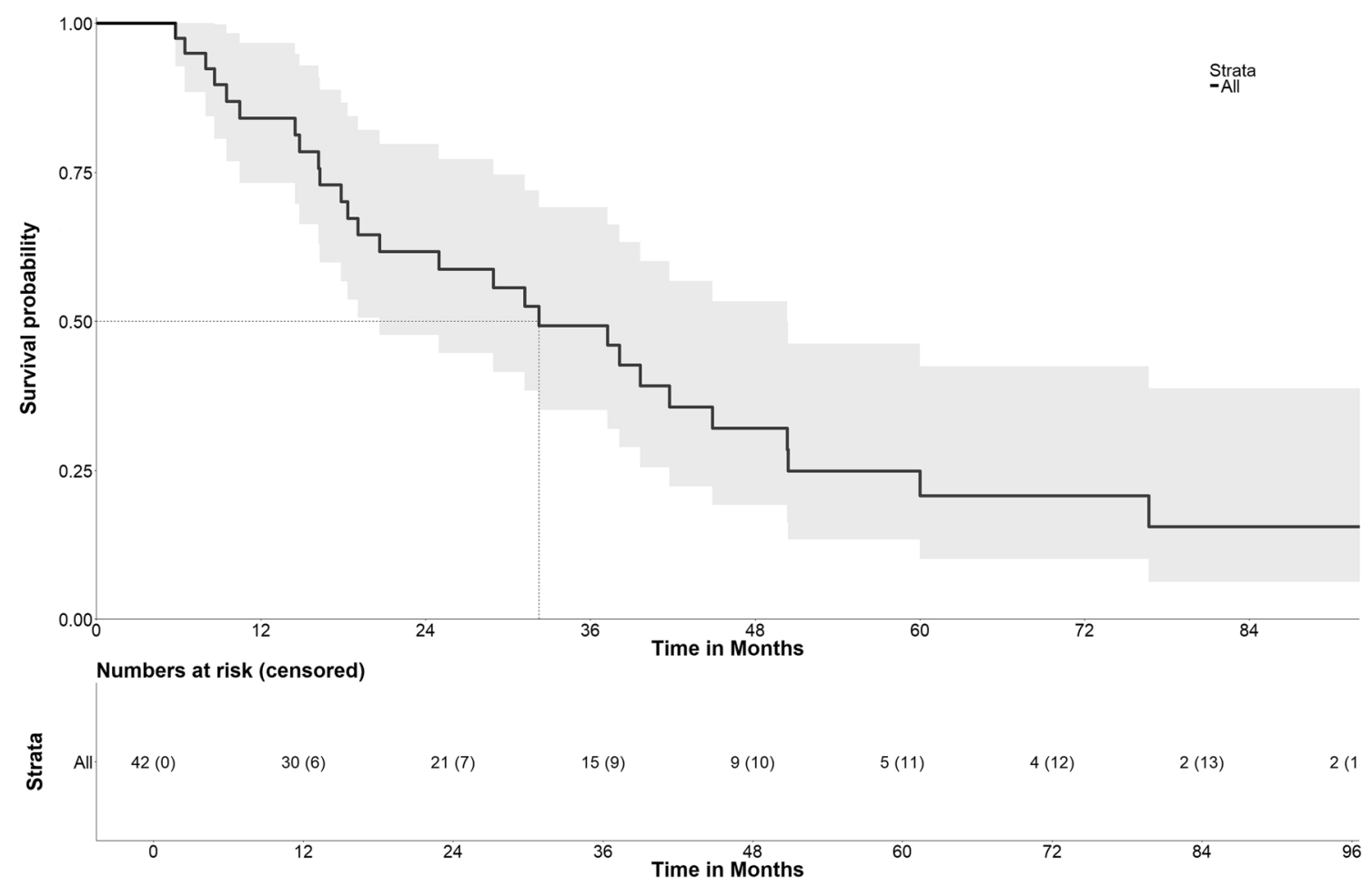

Fig. 2 Overall survival after initial SRFA 


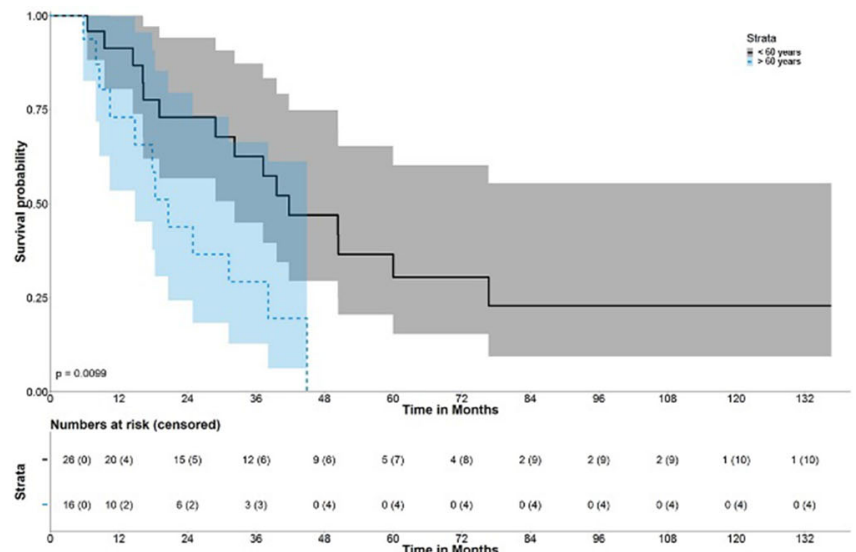

A

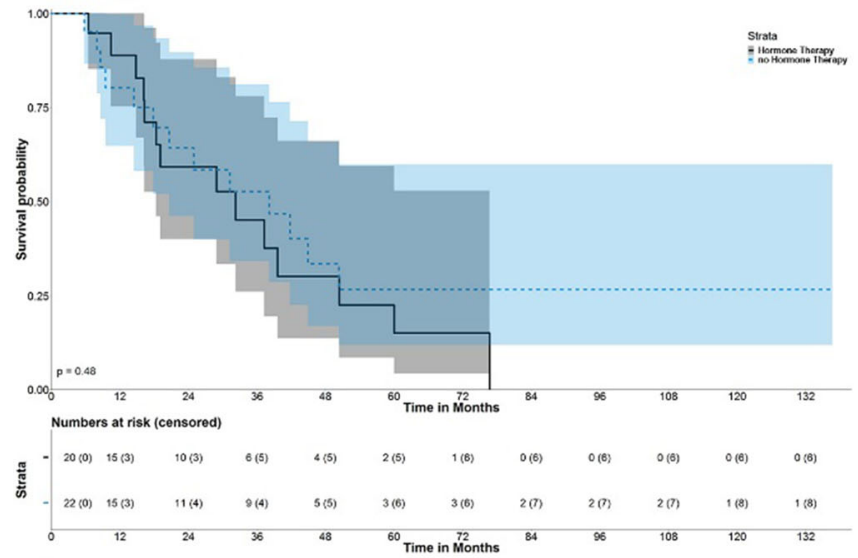

C

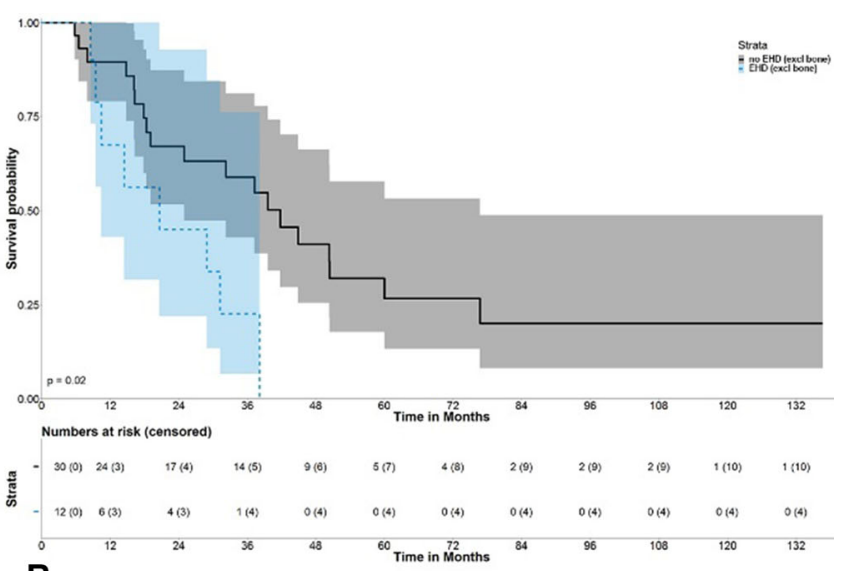

B

Fig. 3 Overall survival after initial SRFA grouped by age (A), extrahepatic disease excluding bone only metastasis (B), hormone therapy (C) and number of tumors (D)

\section{Discussion}

The results of the present study suggest that patients with breast cancer liver metastases benefit from stereotactic radiofrequency ablation (SRFA). More specifically, we found a median OS of 32.2 months from the date of BCLM treatment, which is considerably higher than no treatment, at 3-15 months [20, 21].

In contrast to the substantial evidence behind resection for colorectal liver metastasis, data regarding BCLM are limited. In a systematic review of 33 papers, Fairhurst et al. [22] reported a median OS of 35.1 months and a 5-year survival rate of $33 \%$ after BCLM resection, which is closely aligned with the data of the present study. Newer studies such as Ruiz et al. [23] reported better OS when combining resection with systemic treatment, with a median OS of up to 82 months for liver confined metastases. Regarding survival after conventional RFA in patients with BCLM, several authors showed promising results. The reported median OS rates of several studies range from 26-29.9 months with OS rates at 1,3 and 5 year of $68-90 \%, 25-44 \%$ and $11-27 \%$ [14, 24, 25]. These studies included mainly small tumors up to $3 \mathrm{~cm}$ due to the limitations of conventional targeting techniques. Despite the fact that the majority $(62 \%)$ of patients in the present study had multiple liver metastases with a median size of $3 \mathrm{~cm}$ (up to $9 \mathrm{~cm}$ ), our median OS of 32.2 months, with 1-, 3 - and 5-year OS rates of $84.1 \%, 49.3 \%$ and $20.8 \%$ compare favorably to the literature regarding conventional RFA.

Patient selection, especially in the context of advanced breast cancer patients, is a crucial step towards improving outcomes, which could be improved by identifying prognostic factors associated with better survival. Positive hormone receptor status has been reported as a positive predictive factor for survival after hepatic resection for BCLM [3, 26, 27]. However, we found no evidence for such an association $(p=0.479)$, maybe due to the selected study cohort. In line with our results, Jakobs et al. [28] reported that hormone receptor status did not significantly 
Table 4 Cox regression analyses of factors affecting overall survival (OS) after initial SRFA

\begin{tabular}{|c|c|c|c|c|c|c|c|c|}
\hline \multirow[t]{2}{*}{ Variables } & \multicolumn{3}{|c|}{ OS rates $(\%)$} & \multirow[t]{2}{*}{$\mathrm{OS}_{ \pm}$} & \multirow[t]{2}{*}{ Univariate analyses $p$ value } & \multicolumn{3}{|c|}{ Multivariable analysis } \\
\hline & $1 \mathrm{yr}$ & $3 \mathrm{yr}$ & $5 \mathrm{yr}$ & & & $p$ value & Hazard ratio & $95 \% \mathrm{CI}$ \\
\hline Age & & & & & 0.013* & 0.090 & 2.301 & $0.878-6.031$ \\
\hline$>60 \mathrm{yr}$ old & 73 & 28 & - & 20.6 & & & & \\
\hline$<60$ yr old & 92 & 63 & 30 & 41.8 & & & & \\
\hline Tumor size & & & & & 0.171 & & & \\
\hline$>3 \mathrm{~cm}$ & 92 & 49 & 06 & 32.2 & & & & \\
\hline$\leq 3 \mathrm{~cm}$ & 70 & 51 & 51 & 76.7 & & & & \\
\hline Tumor number & & & & & 0.838 & & & \\
\hline$>1$ & 88 & 46 & 25 & 31.2 & & & & \\
\hline 1 & 79 & 55 & 15 & 38.1 & & & & \\
\hline Tumor distribution & & & & & 0.423 & & & \\
\hline Unilobar & 81 & 51 & 14 & 37.3 & & & & \\
\hline bilobar & 92 & 46 & 36 & 31.2 & & & & \\
\hline Extrahepatic disease & & & & & 0.208 & & & \\
\hline Yes & 82 & 37 & 13 & 28.9 & & & & \\
\hline No & 87 & 57 & 25 & 39.6 & & & & \\
\hline Extrahepatic disease (excluding bone only) & & & & & $0.025 *$ & 0.246 & 1.835 & $0.658-5.120$ \\
\hline Yes & 68 & 23 & 0 & 20.6 & & & & \\
\hline No & 89 & 59 & 27 & 41.8 & & & & \\
\hline Time to BCLM & & & & & 0.129 & & & \\
\hline$<24$ months & 75 & 68 & 37 & 50.4 & & & & \\
\hline$>24$ months & 91 & 35 & 8 & 29.9 & & & & \\
\hline Hormone receptor & & & & & 0.479 & & & \\
\hline Positive & 80 & 52 & 26 & 38.1 & & & & \\
\hline Negative & 89 & 46 & 15 & 32.3 & & & & \\
\hline
\end{tabular}

$p<0.05$ is considered as statistical significance (bold and asterisk)

OS overall survival, \pm median (months), yr year,

affect survival after conventional RFA for BCLM. Late onset of BCLM has also been proposed as a predictor of survival by $\mathrm{He}$ et al. [29] and Hoffman et al. [30] in the surgical literature, although this is again distinct from findings in the RFA literature, including this study. One possible explanation for this difference could be due to the different selection criteria for hepatic resection vs. RFA. Another important reported prognostic factor of survival is the presence of extrahepatic disease at initial therapy, where we found extrahepatic metastases (excluding patients with isolated bone metastases) might be significantly associated with reduced survival $(p=0.025)$, which is an observation supported by Jakobs et al. [28] after conventional RFA.

Several studies $[14,24,31]$ have also shown significantly reduced survival in patients with BLCMs $>2.5 \mathrm{~cm}$ after RFA. Whilst larger tumors were associated with worse survival in our study, this did not reach statistical significance $(p=0.171$, Table 4$)$. In addition, Cox regression analysis revealed age $<60$ years was a positive predictor of survival, which is in line with the results of Dittmar et al. [32] following hepatic resection for BCLM.

Owing to the advantages of our setup, the technical success rate, i.e., accurate needle placement (deviation $\leq 10 \mathrm{~mm}$ from the plan) was $100 \%$. The measurement of the safety margin to determine success represents another important outcome measure, which we have not (yet) used in the present study. However, the importance of an adequate safety margin for local tumor control in patients with colorectal liver metastases and hepatocellular carcinoma has been addressed by our study group recently [33, 34].

Insufficient local control remains a major drawback of conventional RFA, especially for larger tumors with reported local recurrence rates between 14 and 50\% $[14,35]$. As such, to achieve complete ablation in larger tumors, multiple overlapping ablation volumes are required [36], although the resulting increase in complexity using 
multiple needles and pathways is very difficult to achieve with conventional techniques. We have therefore developed a technique which uses sophisticated 3D planning, translation of the plan to the patient using a frameless stereotactic navigation system paired with a neurosurgical aiming device and ablation zone evaluation using image fusion. We recently published a study of 97 patients undergoing SRFA for HCC prior to liver transplantation, and demonstrated complete pathological response in 183 of 188 nodules $(97.3 \%)$, and in 50 of 52 nodules $>3 \mathrm{~cm}$ (96.2\%) [37]. Besides RFA, microwave ablation (MWA) is an important ablative strategy which has a higher, and faster thermal energy transfer [38], which allows for larger ablation zones. However, studies regarding MWA for BCLM are sparse, with small patient numbers and ultimately inconclusive results.

Whilst reported LR rates after conventional RFA for BCLM are between 11.6 and 25\% [14, 24, 25], the majority of lesions are $<3 \mathrm{~cm}$. In comparison, our reported LR rate of $7.3 \%$ compares favorably to these results, given a median tumor size of $3 \mathrm{~cm}$, which we attribute to our scrupulous technique, with the aim of achieving a sufficient ablation margin of at least $5 \mathrm{~mm}$. This is achieved through the use of a sophisticated aiming device with precise needle placement and planning software which also allows fusion with previously acquired MR images in case of poor target visibility. Immediate post-ablation contrast-enhanced CT fusion with planning CT allows rapid and reliable assessment of ablation results with the option of repeat ablation in the same session. This standardized approach to ablation means tumors can be reliably treated without limitation in size [39] (the largest lesion treated in this cohort was $9 \mathrm{~cm}$ ) and number [40]. Finally, in our experience, SRFA is easier to learn than conventional techniques, because important workflows can be trained under laboratory conditions, and the learning curve can be ascended before use in patients.

The mortality and complication rate (Grade $1 \& 2$ ) in our study were $0 \%$ and $10.4 \%(5 / 110)$, respectively, which is considerably higher than reported complication rates for conventional ablation, which range from of 0 to $1.1 \%$ $[14,24,41]$. The explanation for this is very likely to be due to the higher complexity of interventions, whereby tumors are often large and/or multiple. However, 4 out of 5 of the complications were successfully treated in the same anesthetic session and did not change the postoperative course. Nevertheless, our results compare favorably to hepatic resection for BCLM, which confers $0-5.9 \%$ mortality and $15 \%$ major morbidity [22].

\section{Limitations}

The limitations of this study include its retrospective design and a relatively small sample size. This small sample size reduces the accuracy of the subgroup analyses in particular. Use of additional therapies, such as chemotherapy and/or hormone therapy after SRFA should also impact the overall clinical outcome. Furthermore, comparison with previous studies is limited as stereotactic navigation systems were not employed in prior reports.

In conclusion, SRFA is a safe, minimally invasive treatment option in the management of BCLMs for selected patients who might benefit from local treatment, with similar survival rates to hepatic resection.

Author Contributions PS and BR contributed to study concepts, study design and data analysis. PS contributed to statistical analysis and manuscript preparation. DP, GL, GE, YS, CI, PS and BR contributed to data acquisition. DP, GL, GE, YS, CI, EW and BR contributed to manuscript review. EW and PS also involved in manuscript editing.

Funding Open access funding provided by University of Innsbruck and Medical University of Innsbruck. All authors have no conflicts to declare.

\section{Declarations}

Conflict of interest All authors have no conflicts of interest to disclose.

Consent for Publication Consent for publication was obtained for every individual person's data included in the study.

Ethical Approval All procedures performed in studies involving human participants were in accordance with the ethical standards of the institutional and/or national research committee and with the 1964 Helsinki declaration and its later amendments or comparable ethical standards.

Informed Consent Informed consent was obtained from all individual participants included in the study.

Open Access This article is licensed under a Creative Commons Attribution 4.0 International License, which permits use, sharing, adaptation, distribution and reproduction in any medium or format, as long as you give appropriate credit to the original author(s) and the source, provide a link to the Creative Commons licence, and indicate if changes were made. The images or other third party material in this article are included in the article's Creative Commons licence, unless indicated otherwise in a credit line to the material. If material is not included in the article's Creative Commons licence and your intended use is not permitted by statutory regulation or exceeds the permitted use, you will need to obtain permission directly from the copyright holder. To view a copy of this licence, visit http://creativecommons. org/licenses/by/4.0/. 


\section{References}

1. Siegel RL, Miller KD, Jemal A. Cancer statistics, 2018. CA Cancer J Clin. 2018;68(1):7-30.

2. Insa A, Lluch A, Prosper F, Marugan I, Martinez-Agullo A, Garcia-Conde J. Prognostic factors predicting survival from first recurrence in patients with metastatic breast cancer: analysis of 439 patients. Breast Cancer Res Treat. 1999;56(1):67-78.

3. Adam R, Aloia T, Krissat J, et al. Is liver resection justified for patients with hepatic metastases from breast cancer? Ann Surg. 2006;244(6):897-907 (discussion 907-898).

4. Chua TC, Saxena A, Liauw W, Chu F, Morris DL. Hepatic resection for metastatic breast cancer: a systematic review. Eur $\mathrm{J}$ Cancer. 2011;47(15):2282-90.

5. Eng LG, Dawood S, Sopik V, et al. Ten-year survival in women with primary stage IV breast cancer. Breast Cancer Res Treat. 2016;160(1):145-52.

6. Weichselbaum RR, Hellman S. Oligometastases revisited. Nat Rev Clin Oncol. 2011;8(6):378-82.

7. Ruiz A, Castro-Benitez C, Sebagh M, et al. Repeat hepatectomy for breast cancer liver metastases. Ann Surg Oncol. 2015;22(Suppl 3):S1057-1066.

8. Cardoso F, Costa A, Senkus E, et al. 3rd ESO-ESMO international consensus guidelines for advanced breast cancer (ABC 3). Ann Oncol. 2017;28(1):16-33.

9. Lencioni R, Crocetti L. Local-regional treatment of hepatocellular carcinoma. Radiology. 2012;262(1):43-58.

10. Mahnken AH, Pereira PL, de Baere T. Interventional oncologic approaches to liver metastases. Radiology. 2013;266(2):407-30.

11. Cui R, Yu J, Kuang M, Duan F, Liang P. Microwave ablation versus other interventions for hepatocellular carcinoma: a systematic review and meta-analysis. $J$ Cancer Res Ther. 2020;16(2):379-86.

12. Di Martino M, Rompianesi G, Mora-Guzman I, Martin-Perez E, Montalti R, Troisi RI. Systematic review and meta-analysis of local ablative therapies for resectable colorectal liver metastases. Eur J Surg Oncol. 2020;46(5):772-81.

13. Bergenfeldt M, Jensen BV, Skjoldbye B, Nielsen D. Liver resection and local ablation of breast cancer liver metastases-a systematic review. Eur J Surg Oncol. 2011;37(7):549-57.

14. Meloni MF, Andreano A, Laeseke PF, Livraghi T, Sironi S, Lee FT Jr. Breast cancer liver metastases: US-guided percutaneous radiofrequency ablation-intermediate and long-term survival rates. Radiology. 2009;253(3):861-9.

15. Bale R, Widmann G, Stoffner DI. Stereotaxy: breaking the limits of current radiofrequency ablation techniques. Eur $\mathrm{J}$ Radiol. 2010;75(1):32-6.

16. Bale R, Widmann G, Haidu M. Stereotactic radiofrequency ablation. Cardiovasc Intervent Radiol. 2011;34(4):852-6.

17. Bale R, Widmann G, Schullian P, et al. Percutaneous stereotactic radiofrequency ablation of colorectal liver metastases. Eur Radiol. 2012;22(4):930-7.

18. Schullian P, Johnston EW, Putzer D, Eberle G, Laimer G, Bale R. Stereotactic radiofrequency ablation of subcardiac hepatocellular carcinoma: a case-control study. Int $\mathbf{J}$ Hyperthermia. 2019;36(1):876-85.

19. Filippiadis DK, Binkert C, Pellerin O, Hoffmann RT, Krajina A, Pereira PL. Cirse quality assurance document and standards for classification of complications: the cirse classification system. Cardiovasc Intervent Radiol. 2017;40(8):1141-6.

20. BacalbaSa N, Balescu I, Dima S, Popescu I. Long-term survivors after liver resection for breast cancer liver metastases. Anticancer Res. 2015;35(12):6913-7.

21. Rubino A, Doci R, Foteuh JC, et al. Hepatic metastases from breast cancer. Updates Surg. 2010;62(3-4):143-8.
22. Fairhurst K, Leopardi L, Satyadas T, Maddern G. The safety and effectiveness of liver resection for breast cancer liver metastases: a systematic review. Breast. 2016;30:175-84.

23. Ruiz A, van Hillegersberg R, Siesling S, et al. Surgical resection versus systemic therapy for breast cancer liver metastases: results of a European case matched comparison. Eur $\mathrm{J}$ Cancer. 2018;95:1-10.

24. Bai XM, Yang W, Zhang ZY, et al. Long-term outcomes and prognostic analysis of percutaneous radiofrequency ablation in liver metastasis from breast cancer. Int $\mathbf{J}$ Hyperthermia. 2019;35(1):183-93.

25. Veltri A, Gazzera C, Barrera M, et al. Radiofrequency thermal ablation (RFA) of hepatic metastases (METS) from breast cancer (BC): an adjunctive tool in the multimodal treatment of advanced disease. Radiol Med. 2014;119(5):327-33.

26. Abbott DE, Brouquet A, Mittendorf EA, et al. Resection of liver metastases from breast cancer: estrogen receptor status and response to chemotherapy before metastasectomy define outcome. Surgery. 2012;151(5):710-6.

27. Elias D, Maisonnette F, Druet-Cabanac M, et al. An attempt to clarify indications for hepatectomy for liver metastases from breast cancer. Am J Surg. 2003;185(2):158-64.

28. Jakobs TF, Hoffmann RT, Schrader A, et al. CT-guided radiofrequency ablation in patients with hepatic metastases from breast cancer. Cardiovasc Intervent Radiol. 2009;32(1):38-46.

29. He X, Zhang Q, Feng Y, et al. Resection of liver metastases from breast cancer: a multicentre analysis. Clin Transl Oncol. 2020;22(4):512-21.

30. Hoffmann K, Franz C, Hinz U, et al. Liver resection for multimodal treatment of breast cancer metastases: identification of prognostic factors. Ann Surg Oncol. 2010;17(6):1546-54.

31. Kumler I, Parner VK, Tuxen MK, et al. Clinical outcome of percutaneous RF-ablation of non-operable patients with liver metastasis from breast cancer. Radiol Med. 2015;120(6):536-41.

32. Dittmar Y, Altendorf-Hofmann A, Schule S, et al. Liver resection in selected patients with metastatic breast cancer: a single-centre analysis and review of literature. J Cancer Res Clin Oncol. 2013;139(8):1317-25.

33. Laimer G, Jaschke N, Schullian P, et al. Volumetric assessment of the periablational safety margin after thermal ablation of colorectal liver metastases. Eur Radiol. 2021;2021:1-11.

34. Laimer G, Schullian P, Jaschke N, et al. Minimal ablative margin (MAM) assessment with image fusion: an independent predictor for local tumor progression in hepatocellular carcinoma after stereotactic radiofrequency ablation. Eur Radiol. 2020;30(5):2463-72.

35. Sofocleous CT, Nascimento RG, Gonen M, et al. Radiofrequency ablation in the management of liver metastases from breast cancer. Ajr. 2007;189(4):883-9.

36. Chen MH, Wei Y, Yan K, et al. Treatment strategy to optimize radiofrequency ablation for liver malignancies. J Vasc Interv Radiol. 2006;17(4):671-83.

37. Bale R, Schullian P, Eberle G, Putzer D, Zoller H, Schneeberger $S$, et al. Stereotactic radiofrequency ablation of hepatocellular carcinoma: a histopathological study in explanted livers. Hepatology. 2019;70(3):840-50.

38. Poulou LS, Botsa E, Thanou I, Ziakas PD, Thanos L. Percutaneous microwave ablation vs radiofrequency ablation in the treatment of hepatocellular carcinoma. World $\mathrm{J}$ Hepatol. 2015;7(8):1054-63.

39. Schullian P, Johnston EW, Putzer D, Eberle G, Laimer G, Bale R. Safety and efficacy of stereotactic radiofrequency ablation for very large $(>/=8 \mathrm{~cm})$ primary and metastatic liver tumors. Sci Rep. 2020;10(1):1618.

40. Schullian P, Putzer D, Eberle G, Laimer G, Bale R. Simultaneous stereotactic radiofrequency ablation of multiple $(>/=4)$ liver 
tumors: feasibility, safety, and efficacy. J Vasc Interv Radiol. 2020;31(6):943-52.

41. Carrafiello G, Fontana F, Cotta E, et al. Ultrasound-guided thermal radiofrequency ablation (RFA) as an adjunct to systemic chemotherapy for breast cancer liver metastases. Radiol Med. 2011;116(7):1059-66.
Publisher's Note Springer Nature remains neutral with regard to jurisdictional claims in published maps and institutional affiliations. 